the stretch $a$ to the ensuing pause, the second and highest elevation $R$ to the contraction of the apical part of the ventricles, the stretch $\beta$ to the second pause, the elevation $T$ to the contraction of the middle part of the ventricles, and the stretch $\gamma$ to the comparatively long interval in which the whole heart remains at rest until the next beat begins with the contraction of the auricles.

This cardiogram represents the normal heart action of a healthy man. Disease of the heart or any disease that affects the heart, violent exertion, even a change in the manner of breathing, produces changes in the heart's movements, which are accurately recorded in the electro-cardiogram. In these facts reside the great value of the new method of diagnosis, which has been developed especially by Nicolai and Kraus. The condition of the heart is shown by the form of th curre. If the elevations $P$ and $T$ are very low, the heart is weak ; if the point $R$ is lower than the general level marked by the stretches, $a \beta \gamma$, the heart is affected by neurosis; if the point $T$ is directed downward th patient suffers from arteriosclerosis. In the transition from an erect to a reclining posture the curve changes in the manner shown in Fig. 7. Respiration causes the changes illustrated in Fig. 8.

In the electro-cardiogram, therefore, the heart writes its own story, and that of the whole body, so that electro-cardiography is an almost unparalleled method of physiological exploration. Physiology and electrotechnics have an equal share in the development of this new method of research, which presents a striking illustration of the high state of electromedical science.

\title{
A School of Porpoises at the New York Aquarium*
}

\author{
The Difficulties Encountered in Bringing the Catch from Cape Hatteras to New York Alive
}

\section{By C. H. Townsend}

AFTer several discouraging attempts with animals more or less injured, the Aquarium has, not merely a single healthy porpoise, but a school of them. They were received without injuries of any kind, and have already lived in the building much longer than any single injured specimen hitherto received. After five and a half months in a pool 37 foet in diameter and 7 foet and a half months in a pool 37 feet 1 ly the best of condition, feeding, leaping and otherwise disporting themselves after the manner of porpoises on the high seas.

No more popular exhibition of marine life has ever been made in the Aquarium. To have these lively rangers of the open ocean dwelling in our midst is fascinating, and overy citizen who has failod to pay thom vis pects are with wild animals in captivity.

Two previous attempts were made to bring porpoises from Cape Hatteras. Although arrangements for their shipment were perfected, the instructions given were not carried out by those to whom the shipments wer entrusted In the first instance all the animals died bere they were unfortunately shipped dry and could not survive the journey without the cooling and supporting medium of water. The next attempt, made last June, when the same blunder was made, gave only slightly better results. Four of the six porpoises shipped died between Hatteras and Norfolk, Va At the latter point the shipment was met by the Director of the Aquarium, who prompty filled the tank containing the two survivors with water. One of the animals died soon after reaching New York, but the other lived two and a half months, notwithstanding the fact that the heating it had undergone during the first stage of shipment produced numerous festering sores, which eventually ended its career.

Firmly believing that plenty of cool water would insure safety during transportation, the Director of the Aquarium went to Hatteras November 7th, to make sure of the details of shipment which, entrusted to others, had been neglected. As far as the adult animals are concerned, the results have been satisfactory. There are five adults about 8 feet long still living, but the four half-grown porpoises died soon after their arrival in New York. The adults gave no trouble during shipment, while the young were exceedingly restless and continually bruised themselves by their struggles in the shipping tanks.

Porpoises are warm blooded, blubber-covered mammals and give off so much heat that the water of the shipping tanks becomes actually warm, requiring to be replaced by cold water every five or six hours. Immediately after their capture at Hatteras, where they were dragged after their capture at Hatteras, where they were dragged
on the beach with a seine about a thousand feet long, the porpoises were placed for 24 hours in a salt water pond just back of the ocean beach. No chances whatever were taken in the matter of temperature. On the beach their natural heating would no doubt have been

* By permission of the New York Zoological Society. accelerated by the hot sunshine. The following day they were seined out of the pond and placed in the shipping tanks, which were then hoisted on board a schoone and filled with water. During the voyage up Pamlico Sound and even through the Great Dismal Swamp Canal the fresh water in the tanks was changed whenCver it became warm. After reaching the New York

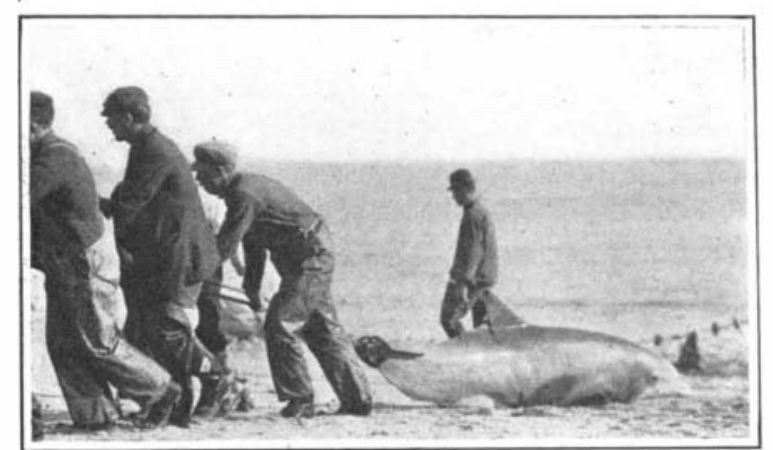

Dragging a porpoise from the net.

steamer at Norfolk the cooling of the porpoise tanks en route was greatly simplified by the use of the salt water hose.

The shipping of porpoises alive is, therefore, a simple matter. Adult animals readily stand transportation while the young animals do not. If carried in long, narrow boxes, just sufficiently large to accommodate them without rubbing, and if kept supplied with sufficient cold water to support and cover them, they can be handled easily enough. There is probably no reason why a porpoise, under such conditions, should not be

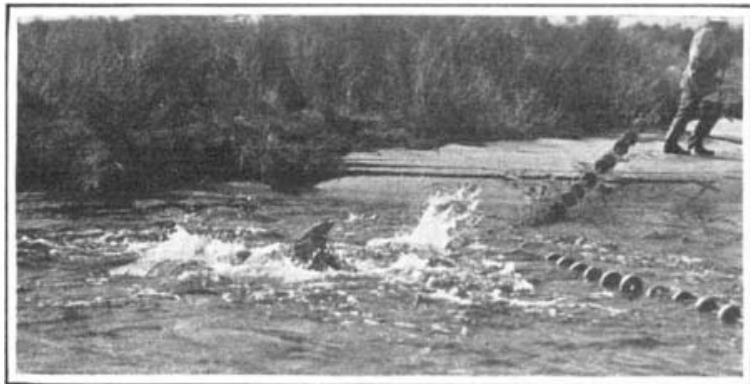

Recapturing the porpoises in the salt-water pond.

carried in a tank many times the distance from Hatteras to New York.

Our porpoises are rather expensive boarders, consuming between 80 and 90 pounds of fresh herring or tomeod a day. For a few days after their arrival they would eat nothing. Within a week they began to take a fow live fishes, and, after having once started to feed, it was not difficult to get them to take dead fish. A few days of hunger brought them around, as it does in the case of the newly captured seal or'sea lion.
Cape Hatteras, singularly enough, is the only point in North America where a porpoise fishery has ever been regularly conducted. The bottle-nosed porpoise appears to winter off our South Atlantic coast and is quite common in the vicinity of Cape Hatteras during the Fall, Winter and Spring months. Schools of porpoises may be seen passing every day just outside the surf. They are taken with a net about one thousand feet long, which is placed a couple of hundred yards outside the line of surf and parallel with it. At each end there is a boat in waiting, ready to carry the haul lines directly ashore as soon as a band of porpoises has passed between the net and the surf. After the lines have been carried ashore, the porpoises are considered fairly secure, for they do not often attempt to cross the lines, and, even when they do, can usually be frightened back by having someone shake and jerk each line continuously. It requires some time to bring the ends of the big seine to the beach, but even then some of the porpoises may get away by leaping over the net or attempting to dive under it. The former can be prevented to some extent by sending a boat to the outer curve of the net, which serves to keep the porpoises from crowding against it. Some of those that attempt to dive underneath become enmeshed, and, being air breathers, are soon drowned. Thirty-three porpoises were beached in the haul of the seine which provided our specimens. Although porpoises have been taken at Cape Hatteras from time mmemorial, the fishery has been conducted in a merely desultory manner, with but little capital invested. The greatest number taken in a single year appears to have been about 1,000 . Porpoises are valuable for their jaw oil, body blubber and hides, the value of each being in the order given. The oil derived from the aws represents the greater part of the value, being worth ordinarily $\$ 25$ a gallon. This oil is extracted from the broad posterior branches of the lower jaw. It is practically the only oil used for the lubrication of watches and similarly delicate mechanisms.

The bottle-nosed porpoise (Tursiops tursio) is the only species of porpoise that has ever been taken at the Hatteras fishery. Our 8-foot specimens represent the average size. A number of specimens were measured in November, however, which exceeded 9 feet in length. The greatest length for this species at Cape Hatteras is 12 feet, but this is altogether unusual. The specimens were presented on the beach at Hatteras by Mr. Joseph $K$. Nye of New Bedford, Mass., the proprietor of the fishery. They were transferred to New York at the expense of the New York Zoological Society.

The porpoise exhibit in the New York Aquarium is absolutely unique. No other aquarium in America or porpoises, and it is doubtful if there are at the present time any other specimens in captivity.

Our bottle-nosed porpoise (Tursiops tursio) closely resembles Delphinus delphis, a species of porpoise or dolphin more abundant in the Eastern Atlantic and in the Mediterranean than along our coast. The latter is

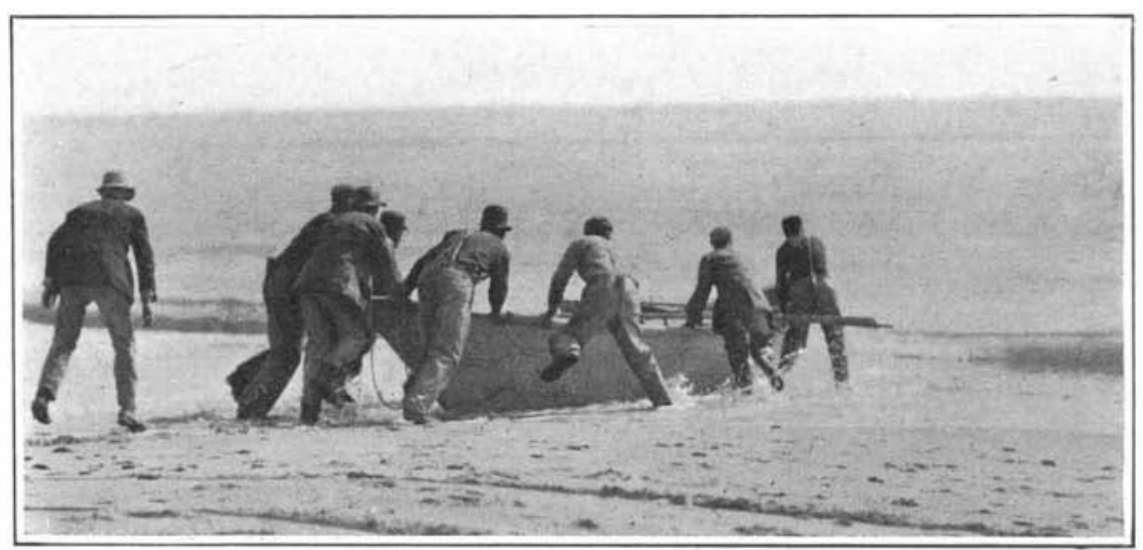

Launching the boat: The porpoises are coming.

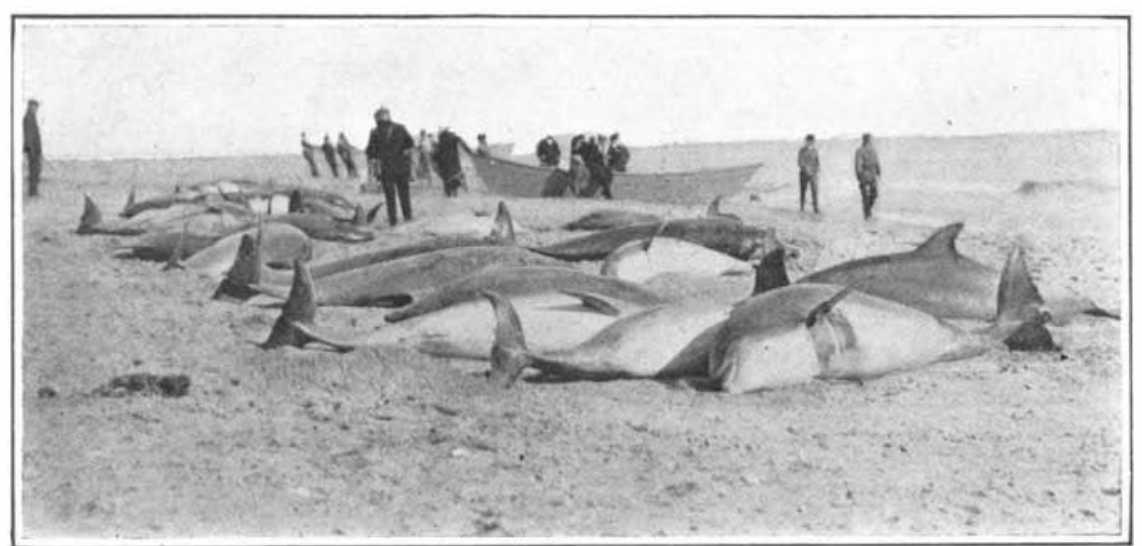

A haul of the porpoise seine, Cape Hatteras, November 12th, 1913. 
the dolphin known to the ancients, which, for some unknown reason, has been systematically caricatured by painters and sculptors since the very beginning of art. Sculptors now have an opportunity to visit the Aquarium and see what the real dolphin looks like.
In the matter of name there is some latitude. All porpoises and dolphins belong to that family of the order of whales called Delphinida, or dolphins, of which there are at least fifty different species, and the names porpoise and dolphin are to some extent interchangeable.
The former is, however, usually applied to the shortjawed kinds. The name "bottle-nose" is inapt in the case of such animals, as the nose or nostrils of all dolphins and porpoises is on top of the head.

The name dolphin is also applied to a fish (Coryphena).

\title{
The Problem of Three Bodies*
}

\author{
A Classic Problem Solved: Its Application to Celestial Bodies Under Mutual Gravitational Attraction
}

\section{By F. R. Moulton}

ONE of the most celebrated problems of mathematics is that of determining the motion of three bodies which attract one another according to the Newtonian law of gravitation. Nearly all of the great mathematicans from Newton to the present time have bestowed upon it their profoundest meditations. Its literature contains memoirs by Euler, Lagrange, Laplace, Poisson, Gauss, Jacobi, Cauchy, Delaunay, Adams, Weierstrass, Poincaré and Darwin, to mention only a few of those who have attempted to penetrate the recondite regions which it includes. These names call to mind many of the finest achievements in mathematics, and the fact that they are all attached to investigations in the same domain proves at once its importance and its difficulty.

The problem of three bodies is of great importance because it is a first step toward the problem of the motions of the members of the solar system under their mutual attractions. The sun, earth, and moon offer an example on which enormous labor has been bestowed, the sun, Jupiter, and Saturn present an example which must be treated by different methods; and triple stars having comparable masses constitute still another case. There are reasons which are obvious to one interested in astronomy why we should be able to predict the positions of the members of the solar system. But the question of its stability, how the orbits of its members have changed in the past, and what alterations they will undergo in the future is from certain points of view much more important. The solution of the problem of three bodies is a prerequisite for a complete and final discussion of the origin of the solar system, its past evolution, and its prospects for the future-questions which have always been of profoundest interest to thinking men.

It is easy to see in a general way the source of some of the difficulty in the problem of three bodies. In order to make the matter perfectly concrete consider the sun, Jupiter, and Saturn, neglecting all the other members of the solar system. As was first proved by the founder of celestial mechanics, Sir Isaac Newton, Jupiter would describe an exact ellipse about the sun as a focus if it were not for Saturn (and the other disturbing bodies) and similarly, Saturn would describe an ellipse about the sun as a focus if it were not for Jupiter. The disturbing attraction of Saturn pulls Jupiter somewhat from its elliptical orbit, and similarly Jupiter pulls Saturn from its orbit. These disturbing forces vary in a complicated fashion because the planets move around the sun at different distances, at different rates and in different planes. The effects of the disturbing forces vary in a still more complicated fashion because they depend not only upon the magnitudes of the forces themselves but also upon the parts of the orbits which the disturbed bodies occupy. In spite of the complexity of this problem it can be solved for such a system as the sun, Jupiter, and Saturn. But the fact that Saturn has been pulled from its undisturbed elliptical orbit makes its pulled from its undisturbed elliptical orbit makes it would otherwise have been. The deviations of Jupiter which have been considered, result in corresponding secondary effects upon its own motion. Similarly, there are secondary effects upon the motion of Saturn due to the fact that both it and Jupiter have departed from thei elliptical orbits by the pnimary perturbations. These secondary effects on the orbits of both bodies give rise to tertiary perturbations upon each. The tertiary effects give rise to those of the fourth order, and so on in an unending series. The deviations increase very rapidly in complexity with their order, and the fact that they soon become numerically less important in such problems as present themselves in the solar system, is the circumstance that has made them tractable by the circumstance that has made them trac
methods that have so far been employed.

The questions that at once arise are whether the problem of three bodies is soluble and whether it has been solved. The purpose of this paper is to answer these questions, so far as they can be answered at the present time, without the use of elaborate mathematical discussions. In a certain sense the answer to both of them is in the affirmative. The answer to the second of these questions is in the affirmative in quite a new sense be cause of a recent remarkable work by Karl F. Sundman

* Reproducod from Popular Astronomy.

1 Research Assoctate of the Carnegie Institution of Washingtion. of Helsingfors, Finland. In a certain theoretical sense his results far surpass any heretofore secured. His whole discussion is of the very highest order of excellence; in fact it is so highly regarded by competent European judges that the distinguished and venerable editor of Acta Mathematica, Prof. Mittag-Leffler, invited him to republish the memoir ${ }^{2}$ in detail in his journal whose pages already were enriched by the researches of Poincaré, Darwin, and Weierstrass. This is another example of the excellent mathematical work which so frequently comes from Finland. Every student of differentia equations and the profounder parts of celestial mechanic has long been familiar with the name of Lindelöff. It shakes one's belief in the justice of the pride in race when he remembers that the Finns are not even of Aryan stock, and of European peoples are related only to the Hungarians. In numbers they are only five or six millions; they are poor and live in a relatively desolate and inhospitable country; they were subject long to Sweden and now to Russia. In spite of all these conditions, ordinarily considered to be the exact opposite of those which are necessary to give lesiure for pursuing the higher things of lif $\theta$, the work which comes from Helsingfors compares most favorably with that produced by many wealthier and more populous peoples.

The present condition of the problem of three bodies will be best understood by giving something of its history. Before taking up the account of its development it should be stated, in order to prevent all misconceptions, that while it is in a certain sense solved, it is by no mean finished. In fact, it has only been begun.

A necessary prerequisite to the treatment of the problem of three bodies was the discovery of the laws of motion and the law of gravitation. These laws were based on an enormous amount of observational experience, from prehistorical antiquity to the culmination in Kepler's laws of the planetary motions. It was from the observations of the motions of the heavenly bodies that men first perceived that there is order in the Universe. This perception lies at the very basis of science, and without it science would not exist. When it was dis covered that apparently the heavenly bodies are moving in an orderly fashion, the next problem was to find th laws of their motions. Among those whose names stand out particularly in connection with the solution of this problem are Hipparchus, Ptolemy, Tycho Brahe, and Kepler. The genius of Newton derived from these laws the law of gravitation.

In order to treat the problem of three bodies it was necessary also to have powerful mathematical processes.
The development of mathematics has been parallel with that of astronomy; in fact, the latter in very many case has forced problems in the former and has often pointed to their solution. But back of particular mathematica theories there lie the logical processes by which they are elaborated. These rules of logic are probably an epitome of the relations among the experiences of the race. A any rate, the logical processes are not contradictory to the Universe about us. In the times of the ancient Greeks all the principal rules of logic were definitely formulated. Certain branches of mathematics wer which alone are sufficient for making progress in the which alone are sufficient for making progress in the problem of three bodies, were initiated by Newton and is but the introduction to the analysis which has grow up in the last two centuries. Those branches of analysis which are particularly valuable in such problems as that of three bodies are especially what is known as the theory of functions of a complex variable and the theory of analytic differential equations based on the theory of functions. Everyone is familiar with the power of the methods of the calculus as compared with those of the more elementary parts of mathematics. The methods of the theory of functions are similarly more powerful than those of the ordinary calculus. It is only by their use that such results as those of Poincaré and Sundman have been secured. The chief difficulty in this paper is to present these highly technical matters in popular terms.

'The work was originally published in Acta Soctetatis Scien matica, vol. 36 (1913).

It should be stated that the writer is not positively informed a
The treatment of the problem of three bodies was begun by Newton in the "Principia." On the basis of his law of gravitation he discussed by synthetic, or geometric, methods the deviations of the moon from elliptic motion which are produced by the disturbing action of the sun. He explained qualitatively all the principal perturbations, and secured quantitative results of considerable approximation. He conceived of the moon as moving in an ellipse whose size, shape, and position continually change. This conception, which grows very naturally out of the ideas connected with the problem of two bodies, has been central in a large part of the perturbation theory. There are grounds for believing that in some respects it has been unfortunate, especially in treating the motion of the moon. The successors of Newton were Clairaut, d'Alembert, and Euler. They developed analysis corresponding to Newton's geometry. Their methods had the generality which is characteristic of analysis, and they were much superior to those of Newton in getting numerical results. But most of their work was devoted to a discussion of the motion of the moon. The methods which they employed were those of successive approximation. The processes were not proved to converge, and therefore no particular properties of the motion of the moon were rigorously established. They were guided, however, by keen physical and geometrical intuitions, and direct observations of the motions of the moon proved that their theories were capable of representing the perturbations with considerable approximation, if not exactly. But theories of this sort, whatever practical value they may have, are not the ones of primary interest in this paper. The center of interest here is on those things which were absolutely proved, with only a secondary interest in their numerical application. In the line of exact results secured by these men mention may be made of the ten integrals of the motion of the problem of three (or any number of) bodies. The problem of three bodies is of the eighteenth order; that is, eighteen integrals are sufficient to solve it completely. Or an equivalent statement is that the complete solution involves eighteen arbitrary constants. Of these eighteen integrals, ten were found by the immediate successors of Newton. Six give the theorem that the center of gravity of the system moves in a straight line with uniform speed; three, that the sums of the products of the masses and the projections on the three reference planes of the rates the respective radii describe areas are constants, and the tenth, that the total energy of the system, both kinetic and potential, is constant. No additional integrals are known for the general case.

The chief immediate successors of Clairaut, d'Alembert, and Euler were Lagrange and Laplace. They perfected the theories of their predecessors and developed corresponding theories for the mutual perturbations of the planets. On the whole their work was marked with greater generality than that which had preceded. One of the most interesting conclusions of a general character, to which both Lagrange and Laplace contributed, was that the major axes of the planetary orbits have no secular terms; that is, they do not on the average increase or decrease. This result was proved only for perturbations of the first order, and even then by breaking up the differential equations in a manner which was not logically justified. Hence, while the conclusion undoubtedly represents the facts closely for a very long time, it was not completely established. The process was not proved to converge and there was no discussion as to what the higher order terms might indicate. The subject was carried somewhat further by Poisson, who proved that so far as the terms of the second order are concerned the conclusions are in a general way the same. Eginitis has found important differences in the terms of the third order, and Poincar6 has proved the process does hot fonverge, so that any conclusions which are drawn ins. it may be erroneous.

Fif far in the discussion no rigorous solutions of the problem of three bodies have been mentioned and no rigorous results, except those which follow from the ten integrals, have been cited. However, in 1772 Lagrange found certain exact solutions of the problem of three bodies. The memoir of Lagrange on this subject has been greatly admired because of the elegance of its form, and the solutions which he proved to exist are very well known. The solutions are characterized by the fact that 